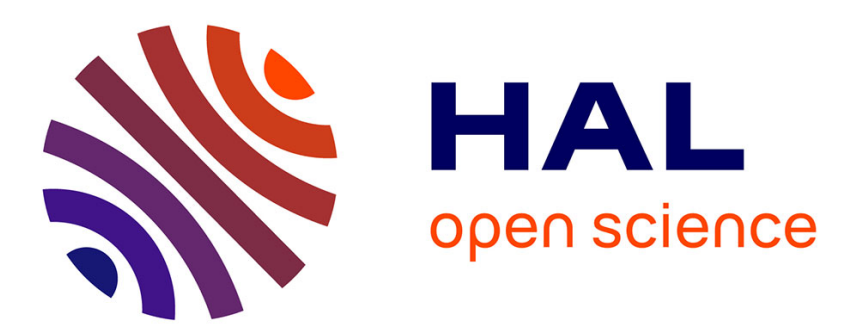

\title{
A quasi-Monte Carlo integration method applied to the computation of the Pollaczek integral
}

Xavier Legrand, Alain Xémard, Gérard Fleury, Philippe Auriol, Carlo Alberto

Nucci

\section{- To cite this version:}

Xavier Legrand, Alain Xémard, Gérard Fleury, Philippe Auriol, Carlo Alberto Nucci. A quasi-Monte Carlo integration method applied to the computation of the Pollaczek integral. IEEE Transactions on Power Delivery, 2008, 23 (3), pp.1527-1534. 10.1109/TPWRD.2007.909050 . hal-00312904

\section{HAL Id: hal-00312904 https://hal.science/hal-00312904}

Submitted on 26 Aug 2008

HAL is a multi-disciplinary open access archive for the deposit and dissemination of scientific research documents, whether they are published or not. The documents may come from teaching and research institutions in France or abroad, or from public or private research centers.
L'archive ouverte pluridisciplinaire HAL, est destinée au dépôt et à la diffusion de documents scientifiques de niveau recherche, publiés ou non, émanant des établissements d'enseignement et de recherche français ou étrangers, des laboratoires publics ou privés. 


\title{
A Quasi-Monte Carlo Integration Method Applied to the Computation of the Pollaczek Integral
}

\author{
Xavier Legrand, Member, IEEE, Alain Xémard, Gérard Fleury, Philippe Auriol, and
}

Carlo Alberto Nucci, Fellow, IEEE

\begin{abstract}
This paper presents an effective numeric method to compute Pollaczek integral. This integral is widely used in transmission-line theory when computing the mutual impedance between an overhead conductor and another overhead or underground conductor and the earth-return impedance of an underground cable. At first, we present mutual expressions proposed by Pollaczek and underline the numerical complexities that often lead to the adoption of alternative simplified methods. Then a brief review of the so called "quasi-Monte Carlo" integration method along with its advantages is given. Such an approach is then applied to compute numerically Pollaczek expressions. Comparison between our numerical procedure and an algorithm that has already been published proves both the procedure accuracy, one of its main advantages along with its ease of implementation, and its relatively low time consumption, independent of the input parameters. Finally, as an application example, quasi-Monte Carlo method is used to assess the adequacy, for several study cases, of the simplified formula by Lucca, which is a widely used approximate expression of interest.
\end{abstract}

Index Terms-Coupled transmission lines, mutual coupling, power cables, power system transients.

\section{INTRODUCTION}

A CLASSICAL approach to simulate the electrical behavior of underground and overhead lines is the so called "transmission line theory" [1]. The first step when using this method is to calculate all mutual and self impedances among conductors. The particular problem of impedance calculation when underground cables are dealt with was treated by Pollaczek in 1926 [2].

Considering the electric field created by infinite thin buried conductors in which a longitudinal current is flowing, Pollaczek presented a formulation for calculating:

- the mutual impedance between two underground conductors;

- the mutual impedance between an underground conductor and an overhead conductor;

- the self ground return impedance of buried conductors.

Manuscript received February 27, 2007; revised May 23, 2007. This work was supported in part by RTE. Paper no. TPWRD-00116-2007.

X. Legrand and A. Xémard are with EDF R\&D, Clamart 92141, France (e-mail: xavier.legrand@edf.fr; alain.xemard@edf.fr).

G. Fleury is with Laboratoire de Mathématiques, Université Blaise Pascal, Clermont-Ferrand 63006, France (e-mail: gerard.fleury@ univ-bpclermont.fr).

P. Auriol is with the AMPERE Laboratory, Écully 69130, France (e-mail: Philippe.Auriol@ec-lyon.fr).

C. A. Nucci is with the Department of Electrical Engineering, University of Bologna, Bologna 40136, Italy (e-mail: carloalberto.nucci@unibo.it).

Digital Object Identifier 10.1109/TPWRD.2007.909050
Pollaczek expressions are given in the form of highly oscillatory integrals hard to compute, so approximations are often preferred in practice to evaluate these impedances [3]-[7].

Despite the existence of these formulae, one can be interested in having an efficient algorithm to compute rigorously Pollaczek expressions in order to:

- evaluate errors due to the use of approximations;

- use such an algorithm instead of direct formulae if it is more suitable in terms of accuracy, computational time, and code implementation complexity.

Few works concern the numerical computation of the rigorous Pollaczek formula. Rearranging it, Uribe obtains in [8] and [9] normalized dimensionless expressions and presented an algorithm to solve them numerically. Such a work is based on a truncation of the infinite integrals, a study of irregularities of the function, and a Simpson's integration method.

In this paper we will follow a different approach. We shall propose a new algorithm to solve numerically the original expressions of the Pollaczek integrals, based on a quasi-Monte Carlo method [10]-[13].

First of all, we will present Pollaczek expressions for their three different cases of application. Then we will give a brief review of the so called "quasi-Monte Carlo" integration method and its advantages before applying it to Pollaczek integrals. A comparative study with results of Uribe's algorithm [8] will allow us to ensure the validity of our approach. Finally, we will focus on Lucca's formula, which is an approximate expression of the mutual impedance between overhead and buried conductors, in order to assess its validity limits.

\section{POLLACZEK EXPRESSIONS}

\section{A. Cases of Interest}

Let us consider the three types of impedances that can be computed with Pollaczek's formula:

- $\mathrm{Z}_{\text {self }}$ is the ground return impedance of an underground cable (Fig. 1, case 1);

- $\mathrm{Z}_{\mathrm{M}-\mathrm{uu}}$ is the mutual impedance between two underground cables (Fig. 1, case 2);

- $\mathrm{Z}_{\mathrm{M}-\mathrm{ua}}$ is the mutual impedance between an underground cable and an aerial line (Fig. 1, case 3).

\section{B. Assumptions}

Pollaczek expressions for $\mathrm{Z}_{\text {self }}, \mathrm{Z}_{\mathrm{M}-\mathrm{uu}}$ and $\mathrm{Z}_{\mathrm{M}-\mathrm{ua}}$ are based on the following assumptions:

- the displacement current is neglected (quasi-static approximation); 


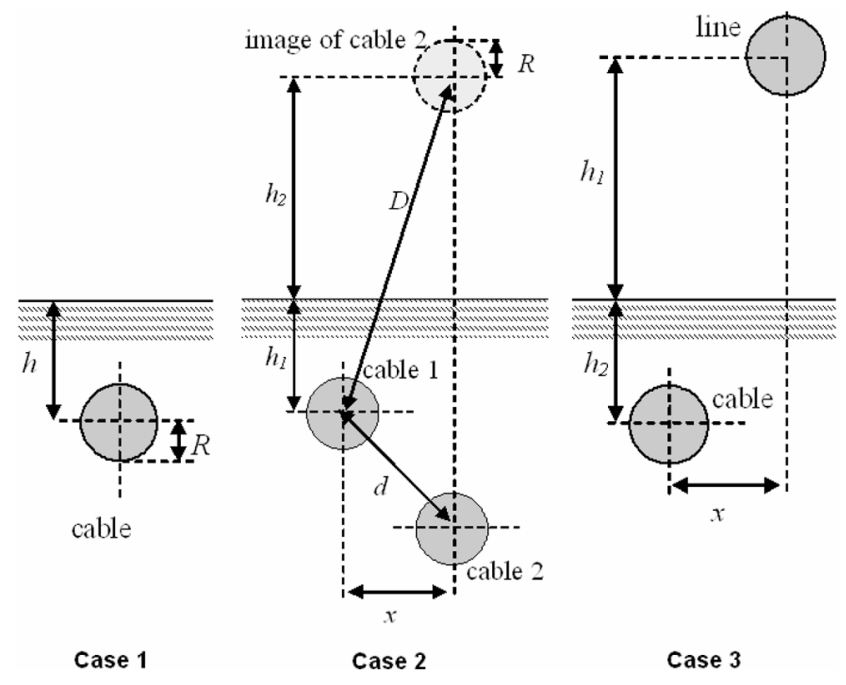

Fig. 1. Cases of interest for the application of the Pollaczek expressions.

- the soil is considered as a semi-infinite homogeneous medium;

- for mutual impedances expressions, conductors are parallel and thin in comparison with their distance;

- all conductors are parallel, infinite and subject to thin wire approximation.

\section{Fundamental Expressions}

Pollaczek gave in [2] the following expressions:

$$
\begin{aligned}
\mathrm{Z}_{\text {self }} & =\frac{j \omega \mu_{0}}{2 \pi} \cdot\left[\mathrm{K}_{0}(R / p)-\mathrm{K}_{0}((2 h) / p)+\mathrm{J}_{\text {self }}\right] \\
\mathrm{Z}_{\mathrm{M} \_ \text {uu }} & =\frac{j \omega \mu_{0}}{2 \pi} \cdot\left[\begin{array}{c}
\mathrm{K}_{0}(d / p)-\mathrm{K}_{0}(D / p) \\
+\mathrm{J}_{\mathrm{M}_{-} \text {uu }}
\end{array}\right] \\
\mathrm{Z}_{\mathrm{M} \_ \text {ua }} & =\frac{j \omega \mu_{0}}{2 \pi} \times\left[\begin{array}{c}
\left.\mathrm{J}_{\mathrm{M}_{-} \text {ua }}\right]
\end{array}\right.
\end{aligned}
$$

where so called "Pollaczek integrals" $\mathrm{J}_{\text {self }}, \mathrm{J}_{\mathrm{M}-\mathrm{uu}}$ and $\mathrm{J}_{\mathrm{M}-\mathrm{ua}}$ are given by

$$
\begin{aligned}
\mathrm{J}_{\text {self }}= & -2 \cdot j \cdot \int_{-\infty}^{+\infty} \frac{\exp \left(-2 h \times \sqrt{\beta^{2}+1 / p^{2}}\right)}{|\beta|+\sqrt{\beta^{2}+1 / p^{2}}} \\
& \cdot \exp (j \beta R) \cdot d \beta \\
\mathrm{J}_{\mathrm{M}_{-} \text {uu }}= & \int_{-\infty}^{+\infty} \frac{\exp \left(-\left(h_{1}+h_{2}\right) \times \sqrt{\beta^{2}+1 / p^{2}}\right)}{|\beta|+\sqrt{\beta^{2}+1 / p^{2}}} \\
& \cdot \exp (j \beta x) \cdot d \beta \\
\mathrm{J}_{\mathrm{M}_{\text {ua }}}= & \int_{-\infty}^{+\infty} \frac{\exp \left(-h_{1} \times|\beta|\right) \cdot \exp \left(-h_{2} \times \sqrt{\beta^{2}+1 / p^{2}}\right)}{|\beta|+\sqrt{\beta^{2}+1 / p^{2}}} \\
& \cdot \exp (j \beta x) \cdot d \beta .
\end{aligned}
$$

The definition of geometric and electrical variables is given in Table I.
TABLE I

NOMENCLATURE

\begin{tabular}{ll}
\hline \hline$j=\sqrt{-1}$ & Complex unit variable; \\
$\omega$ & Angular frequency ; \\
$\mu_{0}=4 \cdot \pi \cdot 10^{-7}$ & Magnetic permeability of vacuum; \\
$p=1 / \sqrt{j \omega \mu_{0} \sigma}$ & Complex depth of the skin effect layer; \\
$\sigma$ & Soil conductivity; \\
$\mathrm{K}_{0}()$ & Modified Bessel function of zero order, \\
$d, D, h, x$ & Second class; \\
\hline \hline
\end{tabular}

TABLE II

DIMENSIONLESS VARIABLES

\begin{tabular}{lll}
\hline \hline \multicolumn{1}{c}{ Case 1 } & \multicolumn{1}{c}{ Case 2 } & Case 3 \\
\hline$\xi=(2 \cdot h) /|p|$ & $\xi=\left(h_{1}+h_{2}\right) /|p|$ & $\xi=h_{2} /|p|$ \\
$\eta=R /(2 . h)$ & $\eta=x /\left(h_{1}+h_{2}\right)$ & $\eta=x / h_{2}$ \\
$\zeta=0$ & $\zeta=0$ & $\zeta=h_{1} / h_{2}$ \\
\hline
\end{tabular}

\section{Dimensionless Expressions}

Pollaczek expressions can be rewritten in term of dimensionless variables $\xi, \eta$ and $\zeta$ [9] defined in Table II.

$$
\begin{aligned}
\mathrm{J}=-2 \times j \times \int_{0}^{\infty} & {\left[\sqrt{u^{2}+j}-u\right] \times \exp \left(-\xi \times \sqrt{u^{2}+j}\right) } \\
& \times \exp (-\xi(\zeta u)) \times \cos (\xi \times \eta \times u) d u
\end{aligned}
$$

with dimensionless variables.

Equation (7) corresponds to Pollaczek's integral. As well known, its numerical integration is difficult because of highly oscillatory terms. In the following sections, we will focus on how to apply a quasi-Monte Carlo method to solve this problem.

\section{Quasi-Monte Carlo Methods to COMPUTE POLLACZEK INTEGRALS}

\section{A. Quasi-Monte Carlo Method}

1) Monte Carlo Integration Method: General idea of Monte Carlo integration method, presented by Metropolis and Ulman in 1949, is to change an integration problem into an average calculation [10].

The strong law of large numbers states that if $\left\{X_{1}, X_{2}, \ldots\right\}$ is an infinite sequence of random variables that are pairwise independent and identically distributed with common expected value $\mathrm{E}\left(X_{i}\right)=\mu$ as $|\mu|<\infty$, then, denoting $\mathrm{P}[\mathrm{X}]$ the probability of an event $X$

$$
\mathrm{P}\left[\left(\lim _{n \rightarrow \infty}\left(\frac{1}{n} \cdot\left(X_{1}+X_{2}+\cdots+X_{n}\right)\right)\right)=\mu\right]=1
$$

i.e., the sample average converges surely to $\mu$.

Let us consider a sequence of $n$ real-valued random variables $X_{i}(1 \leq i \leq n)$ uniformly distributed on interval [0, 
1]. Taking $Y_{i}=f\left(X_{i}\right) \forall i(1 \leq i \leq n)$, with $f$ a real function defined on interval $[0,1]$, we obtain another sequence of $n$ random real-valued independent variables $\left\{Y_{1}, Y_{2}, \ldots, Y_{n}\right\}$ on which we apply the strong law of large numbers

$$
\begin{aligned}
& \mathrm{P}\left[\left(\lim _{n \rightarrow \infty}\left(\frac{1}{n} \cdot\left(Y_{1}+Y_{2}+\cdots+Y_{n}\right)\right)\right)=\mathrm{E}\left(Y_{1}\right)\right]=1 \\
& \mathrm{P}\left[\left(\lim _{n \rightarrow \infty}\left(\frac{1}{n} \cdot\left(\sum_{i=1}^{n} f\left(X_{i}\right)\right)\right)\right)=\mathrm{E}\left(Y_{1}\right)\right]=1 .
\end{aligned}
$$

The expectation of $Y_{1}, \mathrm{E}\left(Y_{1}\right)$ can be written

$$
\left.\mathrm{E}\left(Y_{1}\right)=\int_{0}^{1} f x\right) \cdot d x
$$

Considering $X_{i}$, (10) and (11) lead to

$$
\mathrm{P}\left[\lim _{n \rightarrow \infty}\left(\frac{1}{n} \cdot \sum_{i=1}^{n} f\left(X_{i}\right)\right)=\int_{0}^{1} f(x) \cdot d x\right]=1 .
$$

Taking $n$ large enough, for a particular set $\left\{x^{1}, x^{2}, \ldots, x^{n}\right\}$ of random variables $X_{n}$, the following equality is reasonably verified:

$$
\int_{0}^{1} f(x) \cdot d x \approx \frac{1}{N} \cdot \sum_{i=1}^{n} f\left(x_{i}\right) .
$$

Then the Monte Carlo method consists in approximating integral (11) by a sum, with expression (13). Finally, a confidence interval for the error in the computation can be evaluated as a function of variance $\operatorname{Var}\left(X_{i}\right)$ of the random variables $X_{i}$. However, this interval is probabilistic and gives no absolute upper bound for the error.

2) Quasi-Monte Carlo Method: The main issues in Monte Carlo method are

- the generation of independent uniform random variables. Indeed a inappropriate random source leads to wrong results;

- the probabilistic nature of the error bounding: one cannot be drastically sure of the precision of the results.

Quasi-Monte Carlo simulation uses only sequences of trials $\left\{x^{1}, x^{2}, \ldots, x^{n}\right\}$ having good properties of distribution on the interval $[0,1]$ instead of random numbers as the traditional Monte Carlo method does. Such sequences of numbers are called quasi-random sequences instead of (pseudo) random ones. The discrepancy $D_{n}(x)$ of an $n$ terms sequence reflects the propriety of uniform distribution of its subsequences $\left\{x^{1}, x^{2}, \ldots, x^{i}\right\}(i \in[0, n])$ : the more these subsequences are uniformly distributed and the less is the discrepancy. Therefore, a quasi-random sequence is also called low-discrepancy sequence.

3) Error Bounding: If function $f(x)$ has, according to Hardy and Krause, a bounded variation $V(f)$ on $[0,1]$, then for $\left\{x^{1}, x^{2}, \ldots, x^{n}\right\}$ in $[0,1]$, the so called Koksma-Hlawka inequality will provide an upper bound for the error in quasi-Monte Carlo approximation [14]

$$
\left|\frac{1}{n} \sum_{i=1}^{n} f\left(x^{i}\right)-\int_{0}^{1} f(t) d t\right| \leq V(f) \cdot D_{n}(x)
$$

with $D_{N}(f)$ the discrepancy of the function $f$ and $V(f)$ its variation.

The total variation of function $f$ on $[0,1]$ is

$$
V(f)=\sup \sum_{i=1}^{n-1}\left[\left|f\left(x^{i+1}\right)-f\left(x^{i}\right)\right|\right] .
$$

\section{4) Van Der Corput Sequence:}

a) Definition: The definition of the Van Der Corput sequence was first published in 1935 [15]. It is a low-discrepancy sequence over the unit interval whose terms are obtained as follows: to compute the $i$ th point $x^{i}$ of the Van Der Corput sequence of base $\mathrm{b}$, the integer number $i$ is at first expanded on base $\mathrm{b}$. Then $\mathrm{x}^{\mathrm{n}}$ is obtained by reversing the digits after the "decimal point". For example, let us derive the 12th $(i=12)$ term of the Van Der Corput sequence of base 2:

1) $i$ in base 2 is $i_{b 2}=1100$;

2) after permutation, it becomes: 0011;

3) then in base 10: $x^{n}=0 * 2^{-1}+0 * 2^{-2}+1 * 2^{-3}+1 * 2^{-4}=$ 0.1875 .

Finally, the 12th term of the Van Der Corput sequence of base 2 is 0.1875 .

b) Bounding of the Discrepancy: A useful formula that provides an upper bound to discrepancy $D_{N}(x)$ for a base 2 Van Der Corput sequence is [14]

$$
n \cdot D_{n}\left(\mathrm{C}_{2}\right) \leq \frac{\log _{10}(n)}{3 \times \log _{10}(2)}+1 \quad \forall n \geq 1 .
$$

So (14)-(16) give us an upper bound for the error when using Monte Carlo approximation (13).

5) Advantages of the Quasi-Monte Carlo Method: The main advantages of quasi-Monte Carlo method in the case of interest are:

- the availability of a deterministic bound for error (with the classic Monte Carlo approach, the bound is only probabilistic);

- a faster convergence rate (the error bound decreases to 0 more quickly than $\log _{10}(n) / n$ instead of about $1 / \sqrt{n}$ for classical Monte Carlo);

- the high flexibility (the algorithm does not depend on the function to integrate).

\section{B. Application of Quasi-Monte Carlo Method}

1) The Problem: We present here the application of quasiMonte Carlo integration method to the integration of Pollaczek expression (7). At first, let us factorize integral $\mathrm{J}$ into two terms $\mathrm{J}_{1}$ and $\mathrm{J}_{2}$

$$
\mathrm{J}=-2 \times j \times\left[\mathrm{J}_{1}+\mathrm{J}_{2}\right]
$$


with

$$
\begin{aligned}
\mathrm{J}_{1}= & \int_{0}^{1}\left[\sqrt{u^{2}+j}-u\right] \cdot \exp \left(-\xi \cdot \sqrt{u^{2}+j}\right) \\
& \cdot \exp (-\xi(\zeta u)) d u
\end{aligned}
$$

and

$$
\begin{aligned}
\mathrm{J}_{2}= & \int_{1}^{\infty}\left[\sqrt{u^{2}+j}-u\right] \cdot \exp \left(-\xi \cdot \sqrt{u^{2}+j}\right) \\
& \cdot \exp (-\xi(\zeta u)) d u .
\end{aligned}
$$

We can choose a function $f(u)$ as follows:

$$
\begin{aligned}
& f(u)=\left[\sqrt{u^{2}+j}-u\right] \\
& \cdot \exp \left(-\xi \cdot \sqrt{u^{2}+j}\right) \cdot \exp (-\xi(\zeta u))
\end{aligned}
$$

then

$$
\mathrm{J}_{2}=\int_{0}^{1} f(u) \cdot d u .
$$

To change integration bounds of $\mathrm{J}_{2}$, we use the new variable $v$ defined by

$$
v=\frac{1}{u}
$$

Finally, we obtain a second integral on $[0,1]$

$$
\mathrm{J}_{2}=\int_{0}^{1} f\left(\frac{1}{u}\right) \cdot\left(\frac{1}{u^{2}}\right) d u .
$$

Integrals $\mathrm{J}_{1}$ and $\mathrm{J}_{2}$ can be calculated by quasi-Monte Carlo method in their form (21) and (23).

2) Algorithm Proposed (QMC): It comes from Monte Carlo approximation (13), for $n$ large enough

$$
\begin{aligned}
& \mathrm{J}_{1} \approx \frac{1}{n} \sum_{i=1}^{n}\left[f\left(x^{i}\right)\right] \\
& \mathrm{J}_{2} \approx \frac{1}{n} \sum_{i=1}^{n}\left[f\left(\frac{1}{x^{i}}\right) \cdot\left(\frac{1}{x^{i}}\right)^{2}\right] .
\end{aligned}
$$

So the Pollaczek integral (7) will be calculated by computing the simple expression

$$
J \approx-2 j \cdot \frac{1}{n} \cdot \sum_{i=1}^{n}\left[f\left(x^{i}\right)+f\left(\frac{1}{x^{i}}\right) \cdot\left(\frac{1}{x^{i}}\right)^{2}\right]
$$

where the sequence $\left\{x^{1}, x^{2}, \ldots, x^{n}\right\}$ is the base 2 Van Der Corput sequence of size $n$.

In practice, $f\left(x_{n}\right)$ is computed in the same form as proposed in [9]

$$
\begin{aligned}
f\left(x^{i}\right)=\left[\mathrm{F}\left(x^{i}\right)-\right. & \left.x^{i}+j \cdot \mathrm{G}\left(x^{i}\right)\right] \cdot \exp \left[-\xi \cdot \mathrm{F}\left(x^{i}\right)\right] \\
& \times \exp \left[-j \cdot \xi \cdot \mathrm{G}\left(x^{i}\right)\right] \cdot \cos \left(\xi \cdot \eta \cdot x^{i}\right)
\end{aligned}
$$

with

$$
\mathrm{F}\left(x^{i}\right)=\frac{\sqrt{x^{i_{2}}+\sqrt{x^{i^{4}}+j}}}{\sqrt{2}}
$$

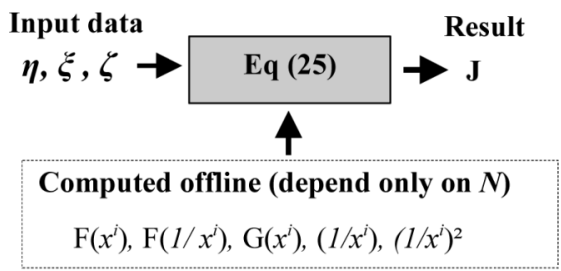

Fig. 2. Quasi-Monte Carlo method applied to the Pollaczek integral calculation.

and

$$
\mathrm{G}\left(x^{i}\right)=\frac{\sqrt{-x^{i_{2}}+\sqrt{x^{i^{4}}+j}}}{\sqrt{2}} .
$$

The terms $\mathrm{F}\left(x^{i}\right), \mathrm{G}\left(x^{i}\right), \mathrm{F}\left(1 / x^{i}\right), \mathrm{G}\left(1 / x^{i}\right)$, and $\left(1 / x^{i}\right)^{2}$ do not depend on geometrical and electrical properties but only on Van Der Corput terms, so they can be computed offline, to reduce calculating time, as shown in Fig. 2.

Finally, (25) is easily computable: its coding is simple and light.

\section{APPLICATIONS}

\section{A. Presentation}

In this section, we will first validate the proposed algorithm by comparing our results with those obtained with the numerical integration method presented in [8] and [9]. Then we will focus on the particular case of the mutual impedance between overhead an underground conductors to present a comparative study with Lucca formula.

We consider here the same minimum and maximum values for electrical and geometrical variables as in [8]

$$
\begin{aligned}
& 4 \mathrm{~m} \leq h_{1} \leq 50 \mathrm{~m} \\
& 0.1 \mathrm{~m} \leq h_{2} \leq 30 \mathrm{~m} \\
& 1 \mathrm{~Hz} \leq f \leq 1 \mathrm{MHz} \\
& 10^{-4} \mathrm{~S} / \mathrm{m} \leq \sigma \leq 1 \mathrm{~S} / \mathrm{m} \\
& 10^{-2} \mathrm{~m} \leq x \leq 10^{3} \mathrm{~m} \\
& 10^{-2} \mathrm{~m} \leq R \leq 10 \mathrm{~m} .
\end{aligned}
$$

It leads to bounds for $|p|$ and normalized dimensionless parameters

$$
\begin{aligned}
& 0.36 \mathrm{~m} \leq|p| \leq 3.6 \cdot 10^{4} \mathrm{~m} \\
& 10^{-4} \leq \xi \leq 10^{2} \\
& 10^{-4} \leq \eta \leq 10^{2} \\
& \zeta=0 \text { or }\left(10^{-1} \leq \zeta \leq 10^{2}\right) .
\end{aligned}
$$

Computing times presented here are obtained on an Intel Xéon $2 \times 3.4 \mathrm{GHz}$ with $4 \mathrm{Go}$ of RAM running Matlab ${ }^{\circledR}$ V6.5.

\section{B. Comparison With Another Numeric Integration Algorithm}

1) Presentation: In [8] and [9], an algorithm is presented to solve dimensionless (7). It is based, as mentioned in the Introduction, on the truncation of infinite Pollaczek's integral and 

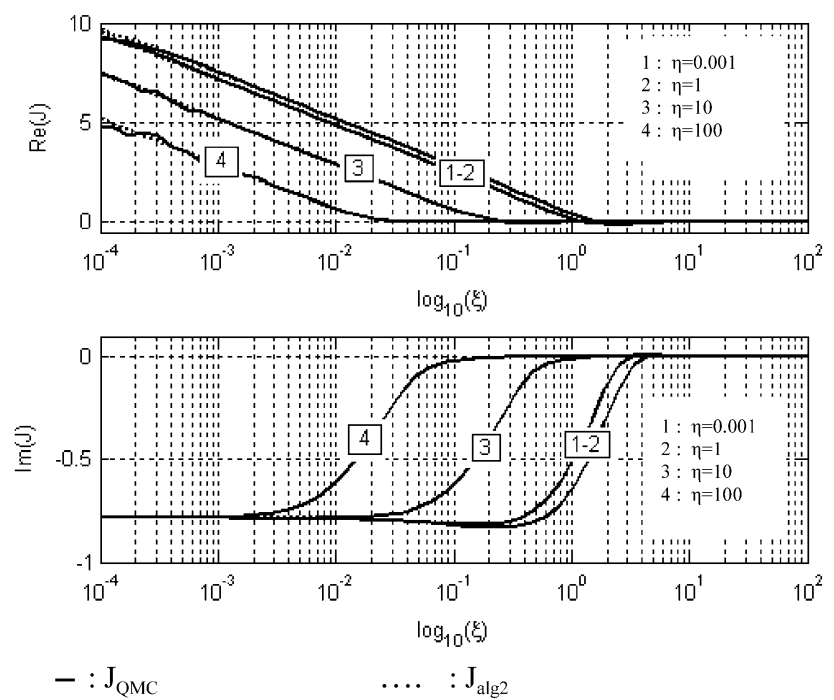

Fig. 3. $\mathrm{J}(\eta, \zeta, 0)$ computed with the two numerical methods.
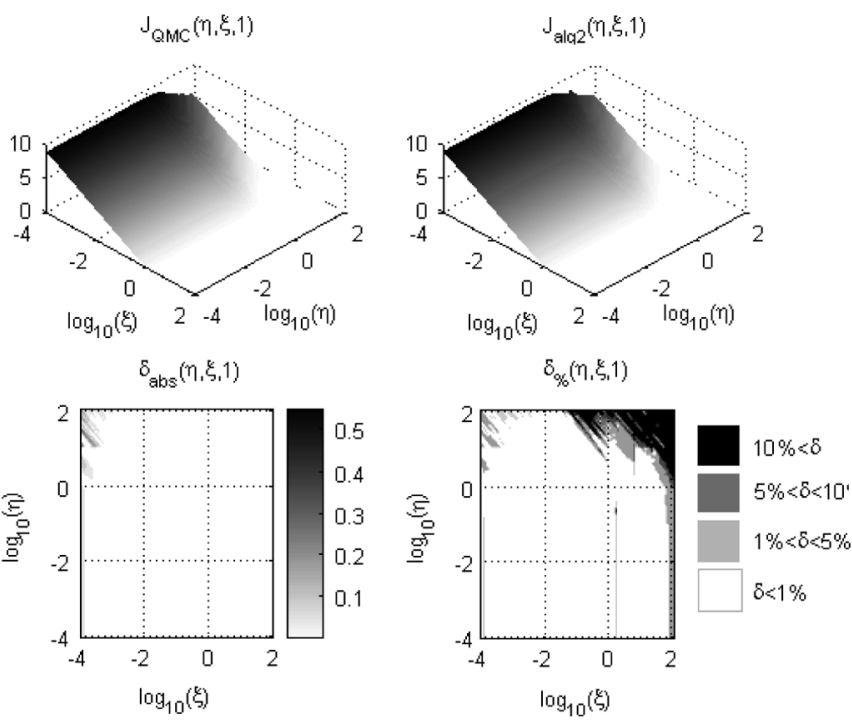

Fig. 4. $\mathrm{J}(\eta, \zeta, 1)$ computed with the two numerical methods, real part.

the study of the integrated function irregularities to adjust a Simpson's method.

2) Comparison of Results: Fig. 3 shows different calculated values of Pollaczek's integral (7), using alternatively the quasiMonte Carlo method presented in this paper $\left(\mathrm{J}_{\mathrm{QMC}}\right)$ and the method presented in [7], [8] $\left(\mathrm{J}_{\mathrm{alg} 2}\right)$. For this work, we have fixed:

- $\zeta=0$, which corresponds to cases 1 and 2 of Fig. 1;

- 10000 integration terms for $\mathrm{J}_{\mathrm{QMC}}$;

- 256 integration terms for $\mathrm{J}_{\mathrm{alg} 2}$.

Fig. 3 shows that the two methods give similar results for $\zeta=0$. Before concluding, we can extend this study for other values of variable $\zeta$. Further, Figs. 4 and 5 present $\mathrm{J}(\eta, \xi, \zeta)$ computed with the two methods for $\zeta=1$. Here, we define absolute and relative differences between the results as follows:

$$
\begin{aligned}
\delta_{\mathrm{abs}}(\eta, \xi, \zeta) & =\left|\mathrm{J}_{\mathrm{QMC}}(\eta, \xi, \zeta)-\mathrm{J}_{\mathrm{alg} 2}(\eta, \xi, \zeta)\right| \\
\delta_{\%}(\eta, \xi, \zeta) & =100 \cdot \delta_{\mathrm{abs}}(\eta, \xi, \zeta) / \mathrm{J}_{\mathrm{QMC}}(\eta, \xi, \zeta)
\end{aligned}
$$
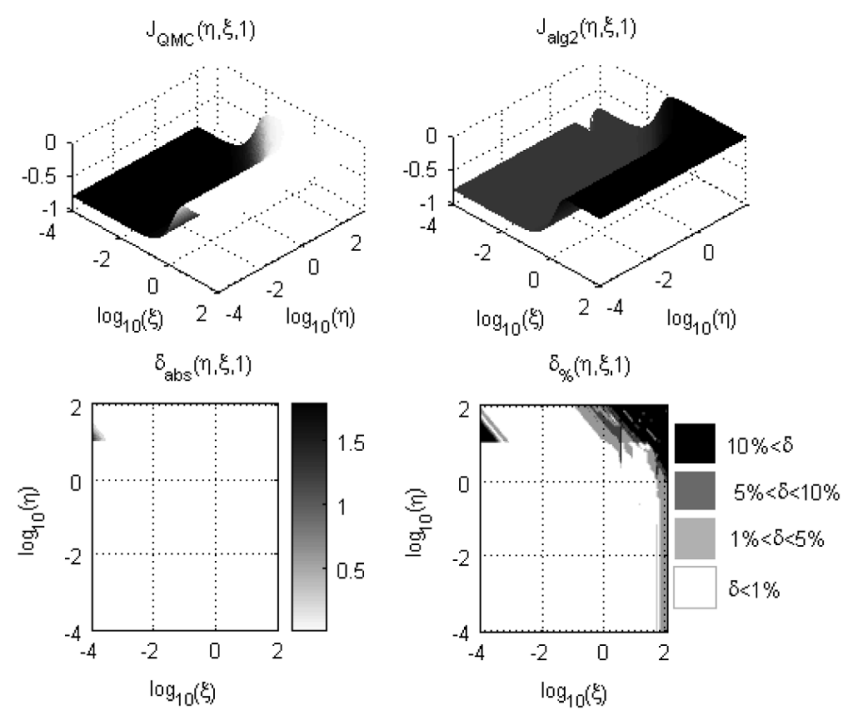

Fig. 5. $\mathrm{J}(\eta, \zeta, 1)$ computed with the two numerical methods, imaginary part.

TABLE III

CASES OF DIFFERENCES BETWEEN THE TwO METHODS

\begin{tabular}{cccc}
\hline \hline $\begin{array}{c}\text { Dimensionless } \\
\text { variables }\end{array}$ & \multicolumn{3}{c}{ Physical variables (cf. Table I) } \\
\hline \hline & Case 1 & Case 2 & Case 3 \\
\hline$\eta>50$ & $h<R / 100$ & $\mathrm{x}>50 \cdot\left(h_{1}+h_{2}\right)$ & $x>50 \cdot h_{2}$ \\
$\&$ & $\&$ & $\&$ & $\&$ \\
$\xi<2 \cdot 10^{-4}$ & $h<10^{-4} \cdot|p|$ & $\left(h_{1}+h_{2}\right)<2 \cdot 10^{-4} \cdot|p|$ & $h_{2}<2 \cdot 10^{-4} \cdot|p|$ \\
& & & \\
\hline \hline
\end{tabular}

These figures can be used to validate our integration algorithm. To this end, it is important to consider the relative difference map $(\delta \%)$ but also to take into account the absolute difference. Indeed for some input data $(\xi>1)$, J tends toward zero and a great value of $\delta \%$ is observed although the difference is insignificant in absolute value $\left(\delta_{\mathrm{abs}}\right)$.

These plots have been completed by others to consider the entire variation domain of dimensionless variables. This work has brought us to the conclusion that both algorithms give results in a good agreement for the great majority of cases. Only a little difference appeared for $\left(\eta>50 \& \xi<2 \cdot 10^{-4}\right)$, which remains on, for each case, to Table III:

For case $1, \eta>50$ leads to the impossible inequality $R>h$ and considering the values of $|p|, \xi<2 \cdot 10^{-4}$ is in practice not likely to be verified for cases 2 and 3; we can thus observe a good agreement between the two algorithms results.

Finally, to illustrate the convergence rate of quasi-Monte Carlo method, we have plotted on Fig. 6, for a practical case and for several values of terms $n$ in (25), $\mathrm{J}_{\mathrm{QMC}}$, the upper bound for the error computed with (14) and $\delta_{\mathrm{abs}}=|| J_{\mathrm{QMC}}|-| \mathrm{J}_{\mathrm{alg} 2}||$.

We consider $h_{1}=h_{2}=3 \mathrm{~m}, f=100 \mathrm{kHz}, \sigma=10^{-2} \mathrm{~S} / \mathrm{m}$, and $x=1 \mathrm{~m}$. It leads to $|p|=11.254 \mathrm{~m}, \xi=0.266, \zeta=1$, and $\eta=1 / 3$.

For this case, $\mathrm{J} \approx 335.55 \cdot 10^{-3}-332.45 \cdot 10^{-3} \cdot j$. If $n>5 \cdot 10^{5}, \delta_{\text {abs }}$ is greater than the upper bound for the error on abs $\left(\mathrm{J}_{\mathrm{QMC}}\right)$, which means that $\mathrm{J}_{\mathrm{QMC}}$ is then more accurate than $\mathrm{J}_{\mathrm{alg} 2}$. Note that this conclusion could be different for other 

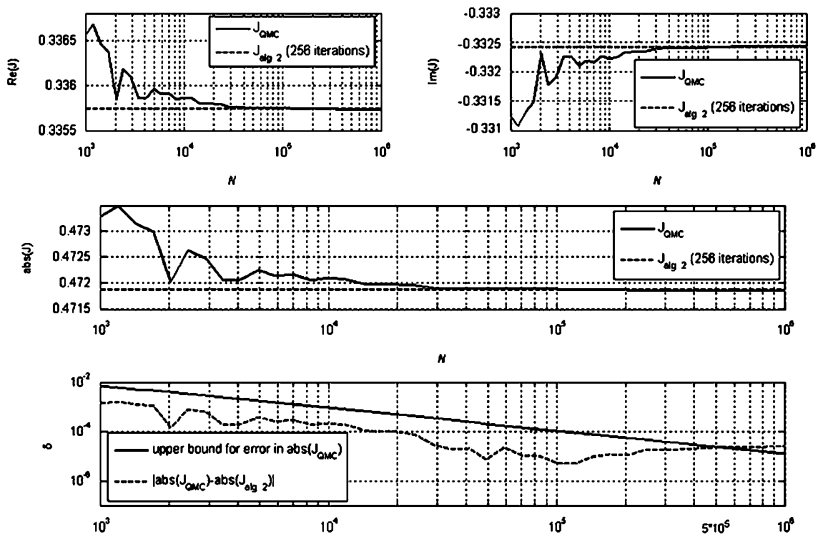

Fig. 6. Particular case of study.

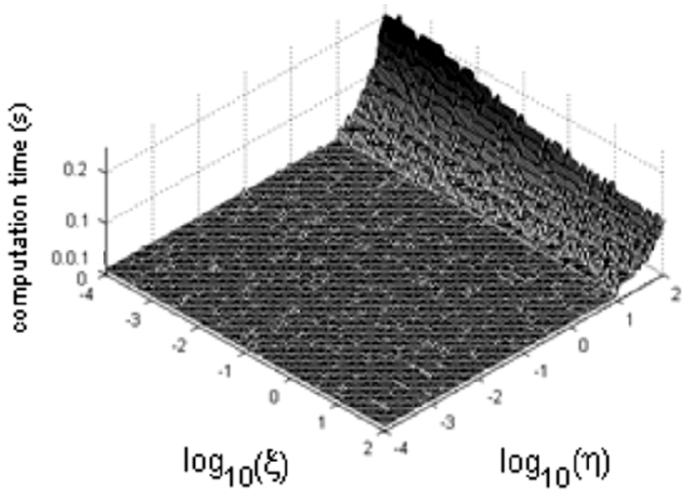

Fig. 7. Computation time for $\mathrm{J}_{\mathrm{alg} 2}(\eta, \zeta, 0)$.

cases, because $\delta_{\text {abs }}$ depends strongly on $\xi, \eta$ and $\zeta$ as has been seen in Figs. 4 and 5.

3) Comparison of Computation Time: Talking about computation time, contrary to the other method, resources needed by the quasi-Monte Carlo algorithm do not depend on the input data. This shows the main advantage of quasi-Monte Carlo method: time consumption does not depend on the behavior of the function to integrate. In our study, to compute an integral value, our algorithm took (with $n=10000$ ), about $16 \mathrm{~ms}$ (this time does not take into account the part "computed offline" shown in Fig. 2).

Considering the algorithm based on a classical Simpson's approach $\left(\mathrm{J}_{\mathrm{alg} 2}\right)$, we have plotted in Fig. 7 the computation time for $\zeta=0$ and different values of $\eta$ and $\xi$ (same computer and 256 integration steps). Although the minimum time is less than the $16 \mathrm{~ms}$ value obtained with quasi-Monte Carlo, the algorithm needs more than $200 \mathrm{~ms}$ for some input data.

4) Comparison of Algorithms Complexity: Because of the very simple shape of (25), quasi-Monte Carlo method leads to an algorithm that is much simpler than the other one considered in which the function to integrate is studied before being computed.
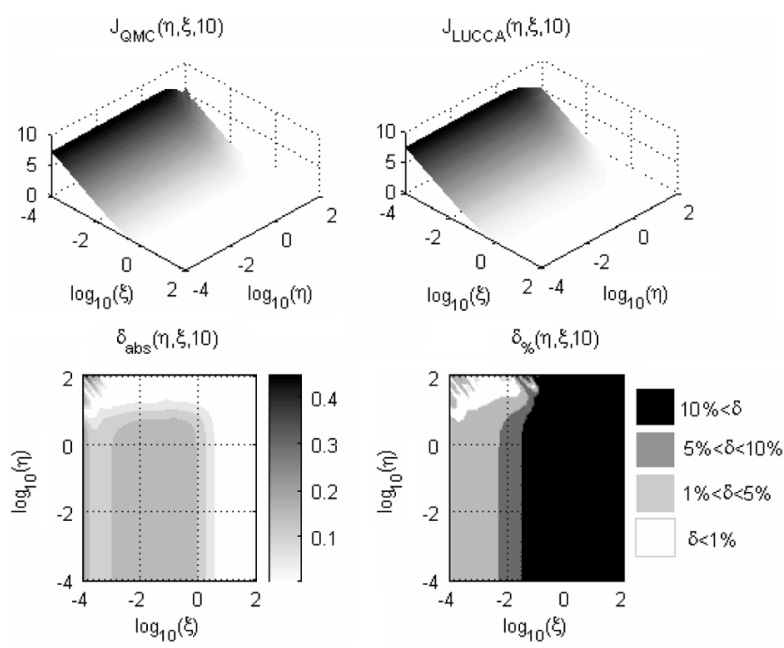

Fig. 8. $\mathrm{J}(\eta, \zeta, 10)$ computed with the quasi-Monte Carlo method and Lucca's formula, real part.

\section{Comparison With the Simplified Formula by Lucca}

1) Presentation: Lucca presented in [3] a closed-form solution for calculating mutual impedances between overhead and buried lines

$$
\begin{aligned}
\mathrm{Z}_{\mathrm{M}-\text { Lucca }}=\frac{j \cdot \omega \cdot \mu_{0}}{2 \pi}\left\{\log \left(\frac{\bar{R}_{12}}{R_{12}}\right)\right. & \\
& \left.-\frac{2 \bar{y}}{2 \gamma^{3}} \cdot\left[\frac{\bar{y}^{2}-3 x^{2}}{\bar{R}_{12}^{6}}\right]\right\}
\end{aligned}
$$

where

$$
\begin{aligned}
R_{12} & =\sqrt{x^{2}+\left(h_{1}-h_{2}\right)^{2}} \\
k_{e}^{2} & =-j \cdot \omega \mu_{0} \sigma \\
\bar{R}_{12} & =\sqrt{\bar{y}+x^{2}} \\
\gamma & =j \cdot k_{e} \text { and } \bar{y}=h_{1}-h_{2}+\frac{2}{\gamma} .
\end{aligned}
$$

The geometrical parameters $h_{1}, h_{2}$ and $x$ are defined in Fig. 1 (case 3).

2) Parametric Study: Figs. 8 and 9 show Pollaczek integral values calculated with our algorithm $\left(\mathrm{J}_{\mathrm{QMC}}\right)$ and with Lucca's formula $\left(\mathrm{J}_{\text {LUCCA }}\right)$ for $\zeta=10$. We present also the absolute and the relative differences between them (respectively, $\delta_{\text {abs }}$ and $\left.\delta_{\%}\right)$.

This study has been carried out for several values of $\zeta$. Maximum errors occur when

$$
-1.5<\log 10(\xi)<1.5 \leftrightarrow 0.03<\log 10(\xi)<30
$$

which can be expressed

$$
0.03|p|<h_{2}<30|p|
$$

Following this approach, one will be able to validate or not the use of Lucca's formula, depending on the accuracy required. 

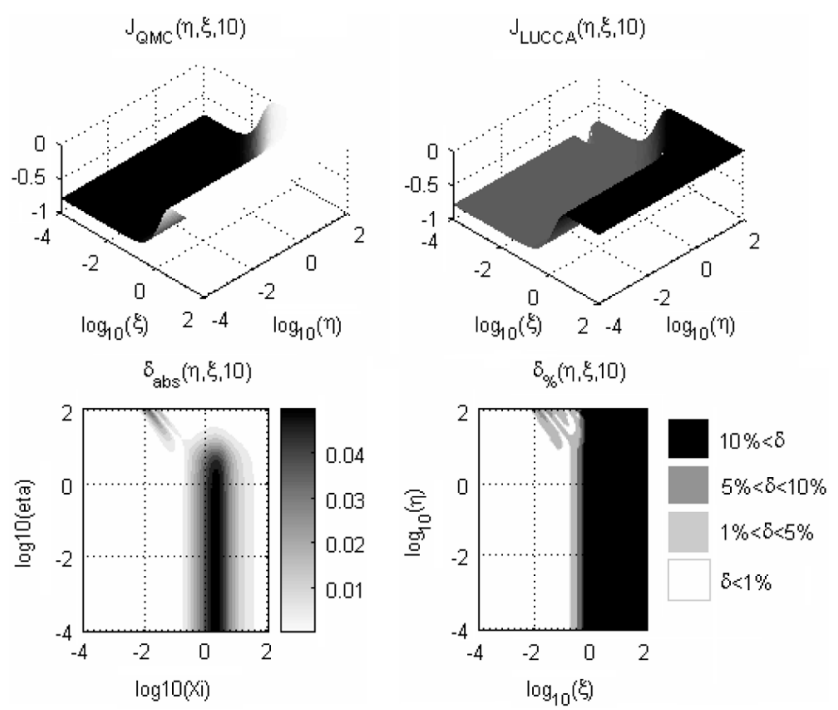

Fig. 9. J $(\eta, \zeta, 10)$ computed with the quasi-Monte Carlo method and Lucca's formula, imaginary part.

TABLE IV

CALCUlation Methods Comparison

\begin{tabular}{lccc}
\hline \hline & QMC & Uribe & Lucca \\
\hline Accuracy & +++ & +++ & + \\
\cline { 1 - 3 }
\end{tabular}

$\begin{array}{llllll}\text { Speed } & \text { highest speed } & + & ++ & & \\ & & & & +++ & ++\rightarrow 3 / 3 \text { (best) } \\ & \text { Stability } & ++ & + & & ++\rightarrow 2 / 3 \\ & & & & & \\ & & & & \end{array}$

\begin{tabular}{lllll}
\hline Algorithm complexity & ++ & + & & +++ \\
\hline \hline
\end{tabular}

\section{CONCLUSION}

As Pollaczek expressions are given in the form of highly oscillatory integrals, hard to compute, approximations are often preferred in practice.

Therefore the availability of an efficient algorithm based on rigorous Pollaczek expressions to check the accuracy of such simplified formulas for the various cases of interest-and to replace them if needed-is of importance.

This paper has proposed and tested a new algorithm in this respect. This algorithm is based on quasi-Monte Carlo integration method with Van Der Corput series. The method that we have proposed has several advantages: it presents a fast convergence rate, an expression to bound the error has been provided, and its computing time is independent on the complexity of the function to integrate. To validate our algorithm, a comparative study with another algorithm has been carried out. This second algorithm, proposed by Uribe in [7], [8] is based on the truncation of the infinite integral, a study of function irregularities and a Simpson's integration method. In general, a good agreement between the results has been obtained. Although the reference algorithm can be faster for some input values, its time consumption - opposite to our method-depends on input data. Furthermore, our algorithm is much simpler to implement.

As an application example, we have focused on the approximate expression of the mutual impedance between overhead and buried conductors provided by Lucca in [3]. Comparing values given by this formula and our approach on the whole electrical and geometrical domain, has allowed us to point out the cases that result in the largest error.

Table IV sum up advantages and drawbacks of the three methods used to calculate the mutual impedance between overhead and buried conductors.

As a last note, we feel worth mentioning that the quasi-Monte Carlo method deserves more attention. For example, it can be relevant to Sommerfeld's integrals computation [12] as well.

\section{REFERENCES}

[1] S. A. Schelkunoff, "The electromagnetic theory of coaxial transmission lines and cylindrical shields," Bell Syst. Tech. J., vol. 13, pp. 532-579, 1934.

[2] F. Pollaczeck, "Uber die induktionswirkungen einer wechelstromeinfachleitung," Elect. Nachrichten, 1926.

[3] G. Lucca, "Mutual impedance between an overhead and a buried line with earth return," Electromagn. Compat., IEE Conf. Publ. 396, 1994.

[4] "Directives concerning the protection of telecommunication lines against harmful effects from electric power and electrified railway lines," Capacitive, Inductive and Conductive Coupling: Physical Theory and Calculation Methods. Geneva, Switzerland, vol. 3, 1989, CCITT, ITU.

[5] O. Saad, G. Gaba, and M. Giroux, "A closed-form approximation for ground return impedance of underground cables," IEEE Trans. Power Del., vol. 11, no. 3, pp. 1536-1545, Jul. 1996.

[6] J.R. Carson, "Wave propagation in overhead wires with ground return," Bell. Syst. Tech. J., vol. 5, pp. 539-554, 1926.

[7] F. Rachidi, S. L. Loyka, C. A. Nucci, and M. Ianoz, "A new expression for the ground transient resistance matrix elements of multiconductor overhead transmission lines," EPSR J., vol. 65, pp. 41-46, 2003.

[8] F. A. Uribe, "Mutual ground impedances between overhead and underground transmission cables," IPST05-151.

[9] F. A. Uribe, J. L. Naredo, P. Moreno, and L. Guardado, "Algorithmic evaluation of underground cable earth impedances," IEEE Trans. Power Del., vol. 19, no. 1, pp. 316-322, Jan. 2004.

[10] N. Metropolis and S. Ulam, "The Monte Carlo method," J. Amer. Statist. Assoc., vol. 44, pp. 335-341, 1949

[11] J. M. Hammersley, "Monte Carlo methods for solving multivariable problems," Ann. New York Acad. Sci., vol. 86, pp. 844-874, 1960.

[12] G. Fleury, F. Paladian, J. Chandezon, and M. Gourgand, "Evaluation numérique d'intégrales de sommerfeld par une méthode quasi Monte Carlo," in Proc. Compatibilité ElectroMagnétique, Toulouse, France, Mar. 2-4, 1994, pp. 317-321.

[13] W. J. Morkoff and R. E. Caflisch, "Quasi-Monte Carlo integration," J. Comput. Phys., vol. 122, no. 2, pp. 218-230, Dec. 1995.

[14] E. Thiémard, "Le calcul et la majoration de la discrépance à l'origine," presented at the l'EPFL, Lausanne, Switzerland, 2000.

[15] J. G. van der Corput, "Verteilungsfunktionen," in Proc. Ned. Akad. vs. Wet., 1935, vol. 38, pp. 813-821.

Xavier Legrand (M'06) was born in France in 1980. He received the M.S. degree in electrical engineering from the Institut National des Sciences Appliquées de Lyon, Lyon, France, in 2004, and is currently pursuing the Ph.D. degree in the research-and-development section of EDF, with the Ampère Laboratory at Lyon.

His research interests include the effects of lightning on aerial and underground transmission lines, grounding systems of lines and substations, and numerical computing of transients in power systems.

Alain Xémard was born in France on December 20, 1961. He graduated from the National Institute of Applied Sciences in Lyon, France, with an engineering degree in electrical engineering in 1985. His research interests include insulation coordination, and development of tools for electromagnetic transient calculation. He has been working at the Research Division of EDF since 1992. He is convener of the CIGRE WG C4 301 dealing with insulation coordination.

Gérard Fleury was born in France in 1951. He received the Ph.D. degree from the University of Clermont-Ferrand, Clermont-Ferrand, France, in 1993.

$\mathrm{He}$ is an Associate with the University Blaise Pascal, Clermont-Ferrand. His research interests include numerical probabilities for engineering. 
Philippe Auriol was born in Beziers, France, in 1947. He received the Engineer degree in electrical engineering from the Institute National Polytechnique de Grenoble, Grenoble, France, in 1970, and the Ph.D. degree in electrical engineering from the University of Lyon, Lyon, in 1977.

He joined the Ecole Centrale de Lyon, Lyon, France, in 1971, and has been full Professor since 1981, and is currently Head of the Electrical Engineering Department of the Ecole Centrale de Lyon. His research activities in the midst of the Ampère Laboratory (CNRS unit no. 5005) include power network transients and high-voltage equipment, lightning phenomena, and electromagnetic compatibility.

Prof. Auriol is a Senior Member of the French SEE, and a Distinguished Member of the CIGRE.
Carlo Alberto Nucci (M'91-SM'02-F'07) was born in Bologna, Italy, in 1956. He received the Electrical Engineering degree (Hons.) from the University of Bologna, Bologna, Italy, in 1982.

Currently, he was a Researcher in the Power Electrical Engineering Institute in 1983, where he became Associate Professor in 1992 and Full Professor, Chair of Power Systems, in 2000. He is author or coauthor of more than 200 scientific papers published in reviewed journals or presented at international conferences. His research interests concern power systems transients and dynamics, with particular reference to lightning impact on power lines, system restoration after blackout, and distributed generation.

Prof. Nucci is a member of the IEEE Working Group "Lightning performance of Distribution lines." He is Chairman of the CIGRE Study Committee C4 "System Technical performance" and is a member of CIGRE Working group C.401 "Lightning," of which he is also Convener. He is also Fellow of the IET. $\mathrm{He}$ is the Chair of the International Steering Committee of the IEEE PowerTech and of the IEEE Power Engineering Society Italian Chapter PE31 in Region 8. Since 2005, he has been Regional Editor for Africa and Europe of the Electric Power System Research Journal. 